\title{
PREVENTION OF SWARMING OF CLOSTRIDIUM SEPTICUM
}

\author{
A. T. Willis and KathleEn Williams \\ Public Health Laboratory, Lewsey Road, Luton
}

\section{Plates XXXII-XXXV}

IN earlier papers (Williams and Willis, 1970; Willis and Williams, 1970; Williams, 1971) we reported that the surface swarming of Clostridium tetani in plate cultures is prevented by incorporation into the medium of commercial tetanus antitoxic serum, and that this technique provides a superior alternative to firm agar for obtaining surface viable counts of $\mathrm{Cl}$. tetani. Because all strains of $\mathrm{Cl}$. tetani are known to share a common $\mathrm{O}$ antigen, it seemed likely that this inhibitory effect on swarming was due to the presence in the antiserum of agglutinating $\mathrm{O}$ antibody to the vegetative cell.

Strains of $\mathrm{Cl}$. septicum are divisible into two groups on the basis of specific components of the $\mathrm{O}$ antigen, neither of which is shared with $\mathrm{Cl}$. chauvoei (Moussa, 1959). Batty and Walker (1963) applied this difference in the $O$ antigenic components of the two organisms to the preparation of fluorescent labelled antibodies which may be used as specific stains to differentiate between Cl. chauvoei and $\mathrm{Cl}$. septicum in smears and in tissue sections.

In the present study we report upon the use of $\mathrm{Cl}$. septicum $\mathrm{O}$ antiserum for the prevention of swarming growth of $\mathrm{Cl}$. septicum in plate cultures.

\section{MATERIALS AND METHODS}

Organisms. Twenty-five strains of $\mathrm{Cl}$. septicum were used. These were kindly provided by Dr S. P. Lapage from the National Collection of Type Cultures (NCTC), and Dr P. D. Walker from the Wellcome Research Laboratory Culture Collection $(\mathrm{CN})$. The strains were respectively nos. NCTC281, 282, 283, 284, 286, 501, 504, 547, 549, 550, 551; and nos. CN424, $2779,3232,3608,3610,3847,3848,3941,3942,3957,4212,5115,5421,5545$. In addition, two strains of $\mathrm{Cl}$. chauvoei (NCTC8070 and 8590) and stock strains of $\mathrm{Cl}$. welchii type A, $\mathrm{Cl}$. bifermentans, $\mathrm{Cl}$. oedematiens type $\mathrm{A}$ and $\mathrm{Cl}$. histolyticum were used.

Preparation of $O$ antigen. This was as described by Batty and Walker.

Preparation of antiserum. The method used was that described by Batty and Walker. Antiserum was prepared against a mixture of four strains of $\mathrm{Cl}$. septicum, each of the two serological groups of Moussa being represented by two strains. The strains used were nos. NCTC501, 551, 281 and 282. Courses of immunisation repeated over a period of months yielded sera with agglutinating titres of the order of 2560 .

Media. Fresh horse blood agar was used as the plating medium, and was prepared in the usual way from an ox heart digest broth base (Cruickshank, 1965); layer plates containing 5 per cent. of defibrinated horse blood (Wellcome) were used. The ordinary agar medium contained 1.5 per cent. of New Zealand agar; firm agar plates contained 4 per cent. of New Zealand agar. For the plate culture of $\mathrm{Cl}$. chauvoei the medium was enriched with

Received 25 Feb. 1972; accepted 24 March 1972.

J. MED. MICROBIOL.-VOL. 5 (1972) 
0.5 per cent. of glucose and 1 per cent. of liver extract. All media contained 0.05 per cent. of cysteine hydrochloride. $\mathrm{Cl}$. septicum antiserum blood agar was prepared by spreading the blood agar plates with $0 \cdot 2-0.5 \mathrm{ml}$ of the undiluted rabbit serum.

Seeding of plates. In order to assess inhibition of swarming growth, half-antiserum plates were seeded in a chordal area across the centre of the plate at right angles to the antiserum area. Surface viable counts were performed by the method of Miles, Misra and Irwin (1938) with 10-fold dilutions of the inoculum prepared in 0.9 per cent. $\mathrm{NaCl}$ in water containing 0.05 per cent. of cysteine hydrochloride (BDH). Cultures were incubated for 24 and $48 \mathrm{hr}$ in an anaerobic atmosphere containing 10 per cent. $\mathrm{CO}_{2}$. In all cases the inoculum was the supernatant from a fresh 18-hr cooked meat broth culture of the organism under test.

TABLE

Surface viable counts and type of growth obtained with ten strains of Clostridium septicum on antiserum blood agar and firm blood agar

\begin{tabular}{c|c|c}
\hline \multirow{2}{*}{$\begin{array}{c}\text { Strain of } \\
\text { Cl. septicum }\end{array}$} & \multicolumn{2}{|c}{ Surface viable count per ml on } \\
\cline { 2 - 3 } & antiserum blood agar & firm blood agar \\
\hline NCTC281 & $10^{8} \mathrm{D}^{*}$ & \\
NCTC501 & $2 \cdot 5 \times 10^{7} \mathrm{D}$ & $10^{8} \mathrm{R} \dagger$ \\
CN3847 & $4 \times 10^{8} \mathrm{D}$ & $5 \times 10^{7} \mathrm{R}$ \\
CN3848 & $1 \cdot 5 \times 10^{6} \mathrm{D}$ & $4 \times 10^{8} \mathrm{R}$ \\
CN3941 & $10^{8} \mathrm{D}$ & $\mathrm{RNCP}$ \\
CN3942 & $2 \times 10^{8} \mathrm{D}$ & $108 \mathrm{R}$ \\
CN3957 & $2 \cdot 5 \times 10^{-} \mathrm{D}$ & $3.5 \times 10^{8} \mathrm{R}$ \\
CN4212 & $1.5 \times 10^{9} \mathrm{D}$ & $1.5 \times 10^{6} \mathrm{R}$ \\
CN5115 & $10^{7} \mathrm{D}$ & $\mathrm{RNCP}$ \\
CN5545 & $10^{7} \mathrm{D}$ & $1.3 \times 10^{7} \mathrm{R}$ \\
& & $10^{8} \mathrm{R}$ \\
\hline
\end{tabular}

* $\mathrm{D}=$ Discrete colonies; $\uparrow \mathrm{R}=$ rhizoidal colonies; $¥ \mathrm{RNCP}=$ rhizoidal, no count possible.

RESULTS

Inhibition of swarming growth of Clostridium septicum by antiserum

All 25 strains of $\mathrm{Cl}$. septicum showed complete inhibition of swarming growth on the antiserum half of the plate. Colonies and areas of confluent growth of $\mathrm{Cl}$. septicum on the antiserum medium showed an irregular edge, but this rhizoidal structure was much more truncated than is usually seen on ordinary blood agar. Discrete colonies showed a particularly compact structure, commonly presenting an entire appearance to the naked eye; under the plate microscope these colonies showed a mildly lobulated edge. As expected, most strains showed haemolytic activity which was not inhibited by the antiserum (figs. 1 and 2).

Comparison of surface viable counts on antiserum blood agar and firm blood agar

The surface viable counts of ten strains of $\mathrm{Cl}$. septicum (including two of the strains used in the preparation of the antiserum), performed on firm blood agar and on antiserum blood agar, are summarised in the table; they represent the mean of quadruplicate counts in each case. None of the strains produced overt swarming on either of these media. 
Prevention of SWARMing of CL. SEPTICUM

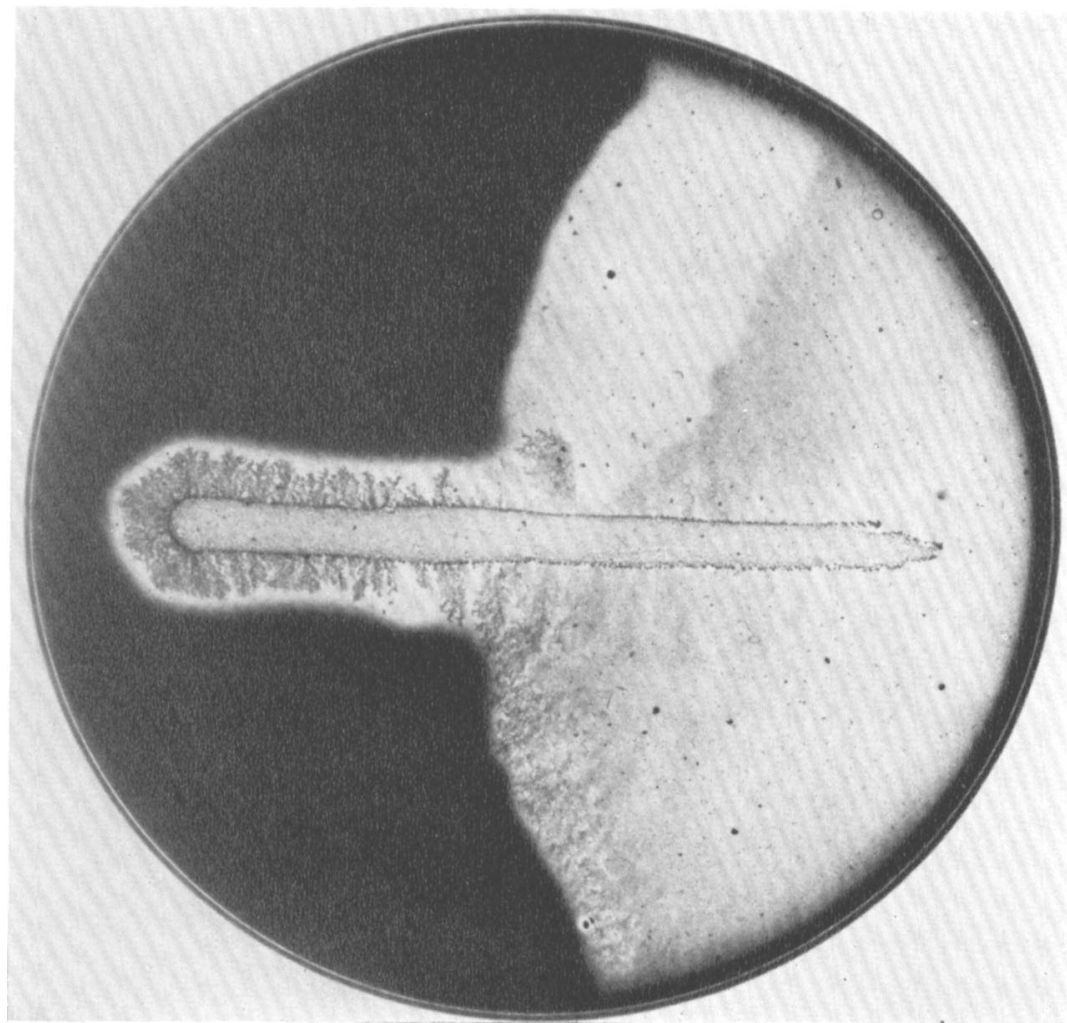

FIG. 1.-Cl. septicum on half-antiserum horse blood agar. The swarming growth is inhibited by Cl. septicum antiserum on the left-hand half of the plate. Haemolytic activity is not affected. $\times 1 \cdot 2$.

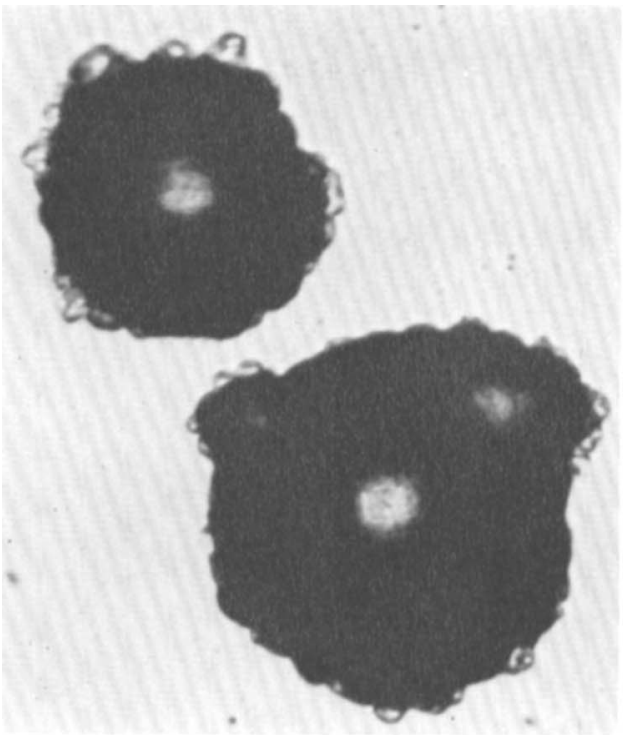

FIG. 2.-Discrete colonies of Cl. septicum on antiserum-treated horse blood agar. $\times 72$.

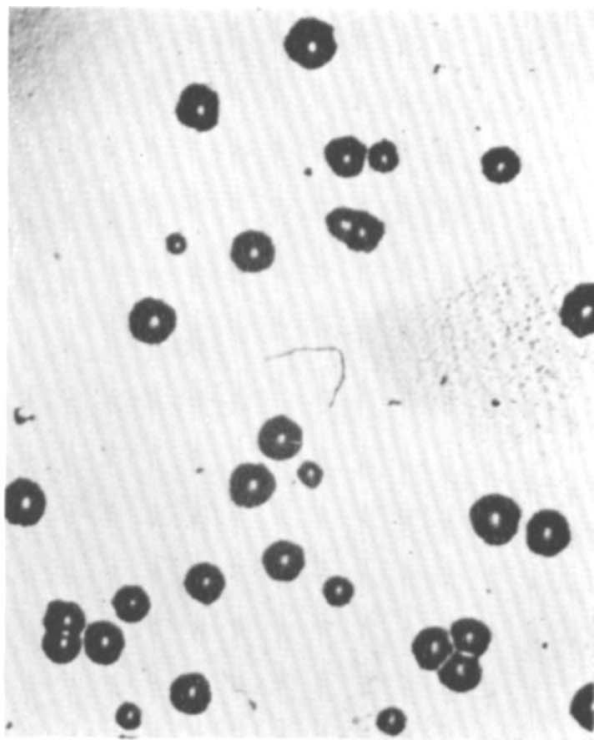

FIG. 3.-Discrete colonies of $\mathrm{Cl}$. septicum in a surface viable count inoculation zone on $\mathrm{Cl}$. septicum antiserum horse blood agar. $\times 14$. 
Prevention of SWARMing of CL. Septicum

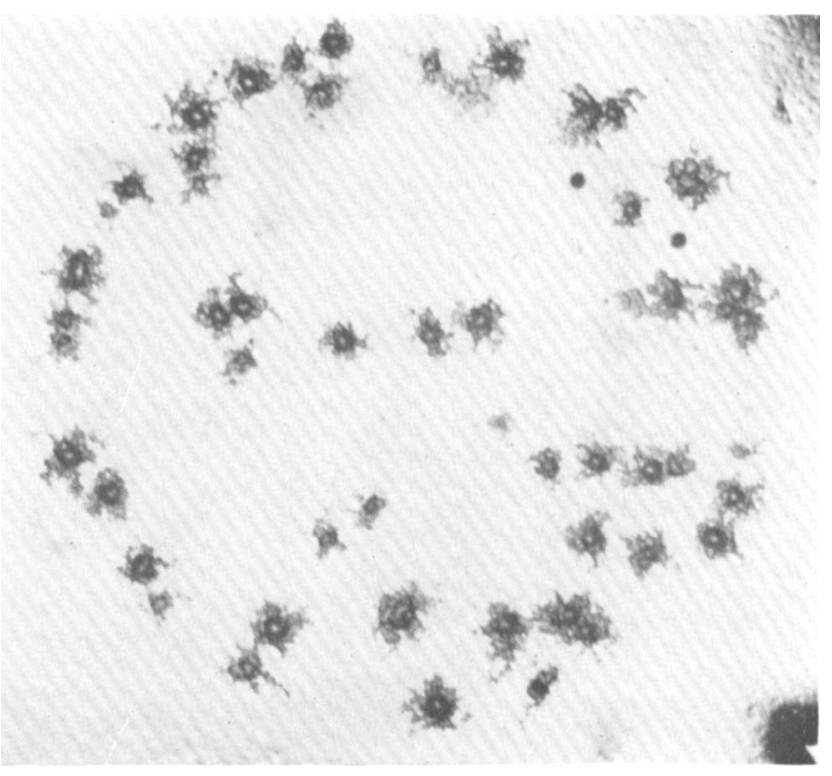

FIG. 4.-Rhizoidal colonies of $\mathrm{Cl}$. septicum in a surface viable count inoculation zone on firm (4 per cent.) agar. $\times 14$.

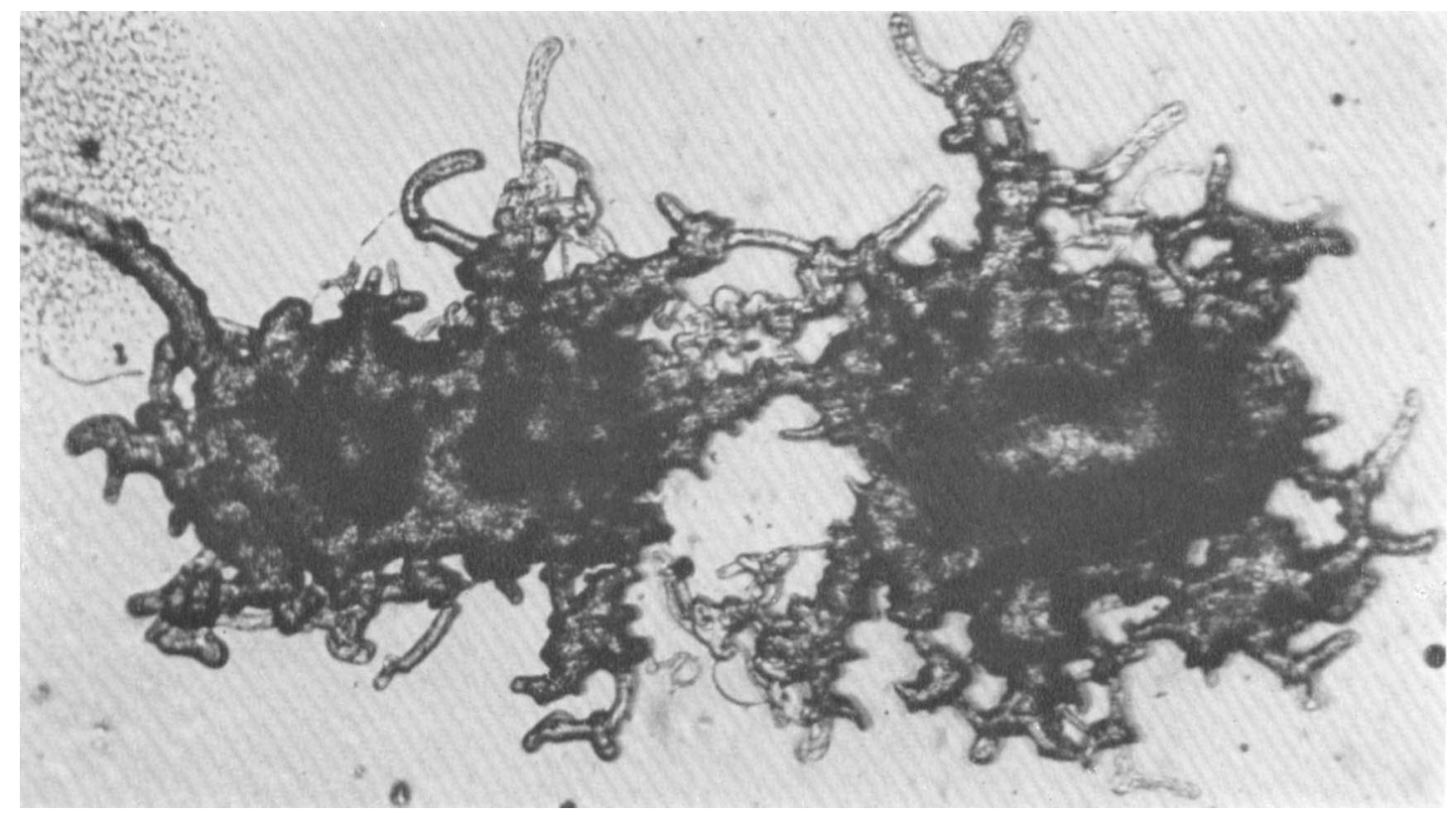

FIG. 5.-Higher-power view of two of the colonies shown in fig. $4 . \quad \times 72$. 
Prevention of SWarming of $C L$. Septicum

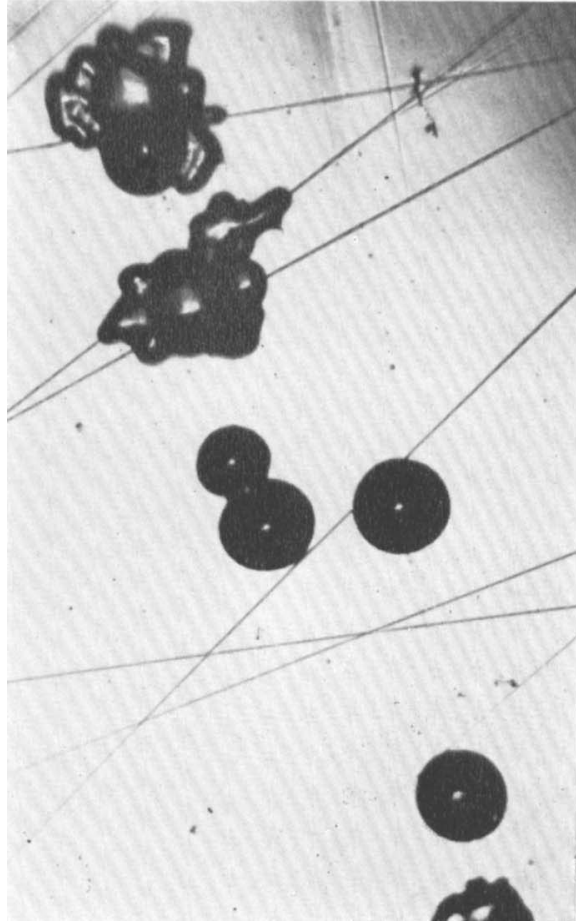

FIG. 6.-Discrete colonies of Cl. septicum and Cl. chauvoei on $\mathrm{Cl}$. septicum antiserum horse blood agar. $\times 36$.

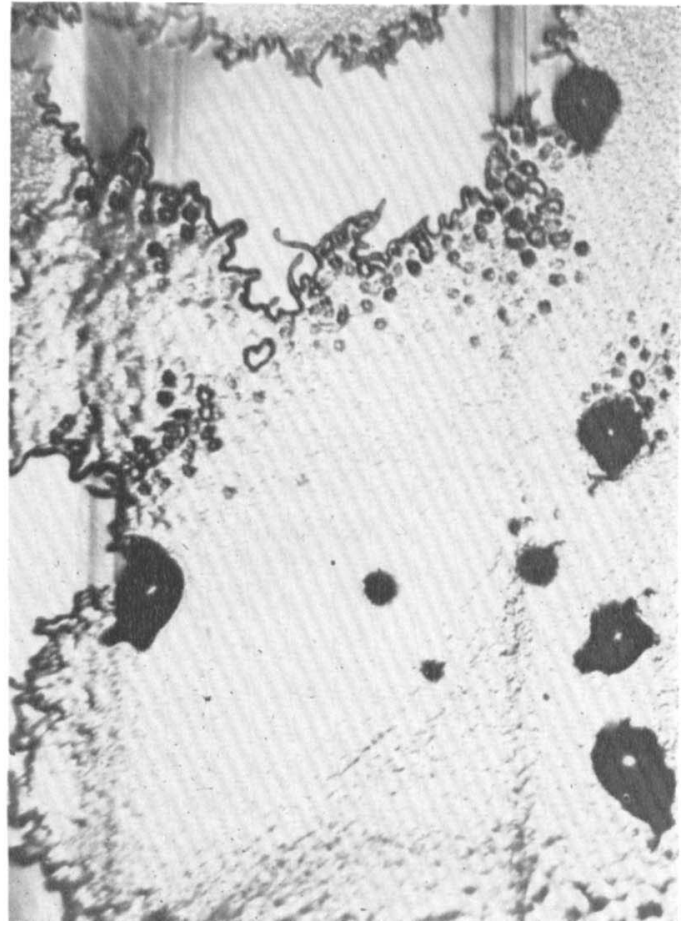

FIG. 7.- Colonies of $\mathrm{Cl}$. chauvoei overgrown by swarming growth of $\mathrm{Cl}$. septicum on horse blood agar. $\times 36$.

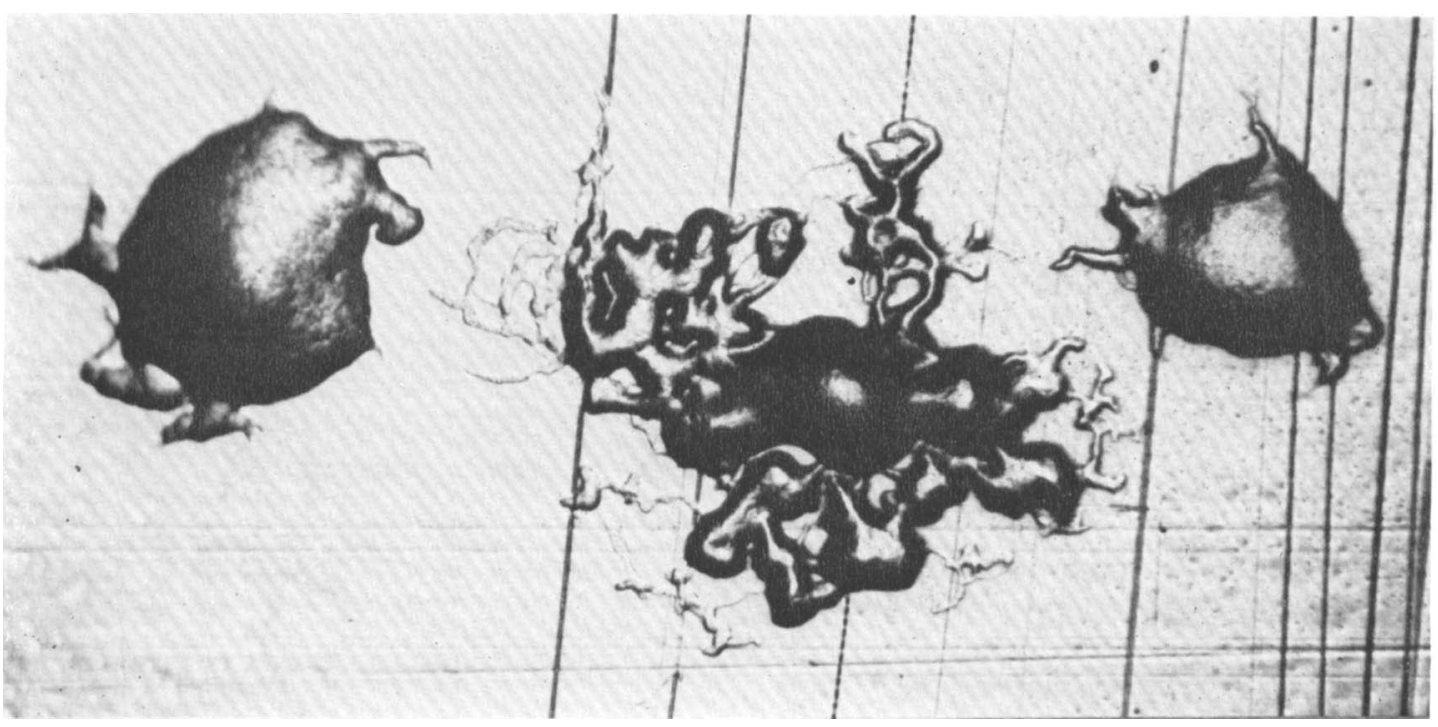

FIG. 8.-Colonies of $\mathrm{Cl}$. oedematiens type A on either side of a discrete colony of $\mathrm{Cl}$. septicum on Cl. septicum antiserum horse blood agar. $\times 72$. 


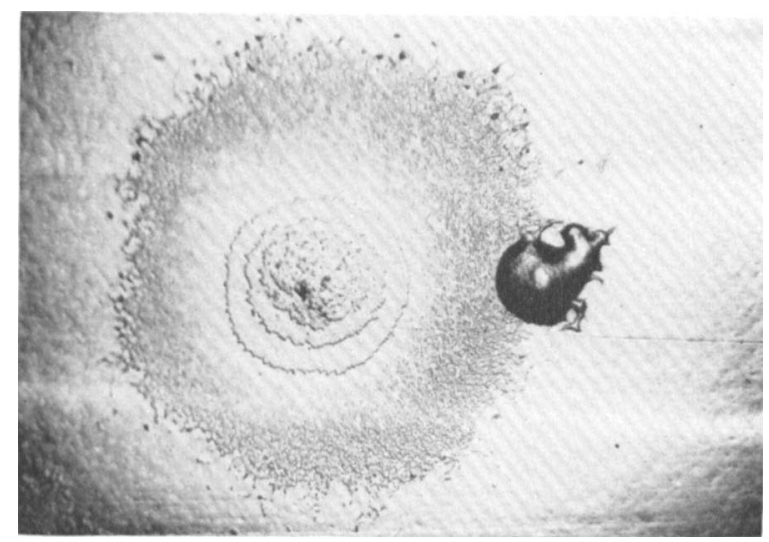

Fig. 9.-Colony of Cl.oedematiens type A being engulfed by swarming growth of $\mathrm{Cl}$. septicum on horse blood agar. $\times 18$.

FIG. 10.-Discrete colonies of Cl. welchii and $\mathrm{Cl}$. septicum on $\mathrm{Cl}$. septicum antiserum horse blood agar. $\times 36$.
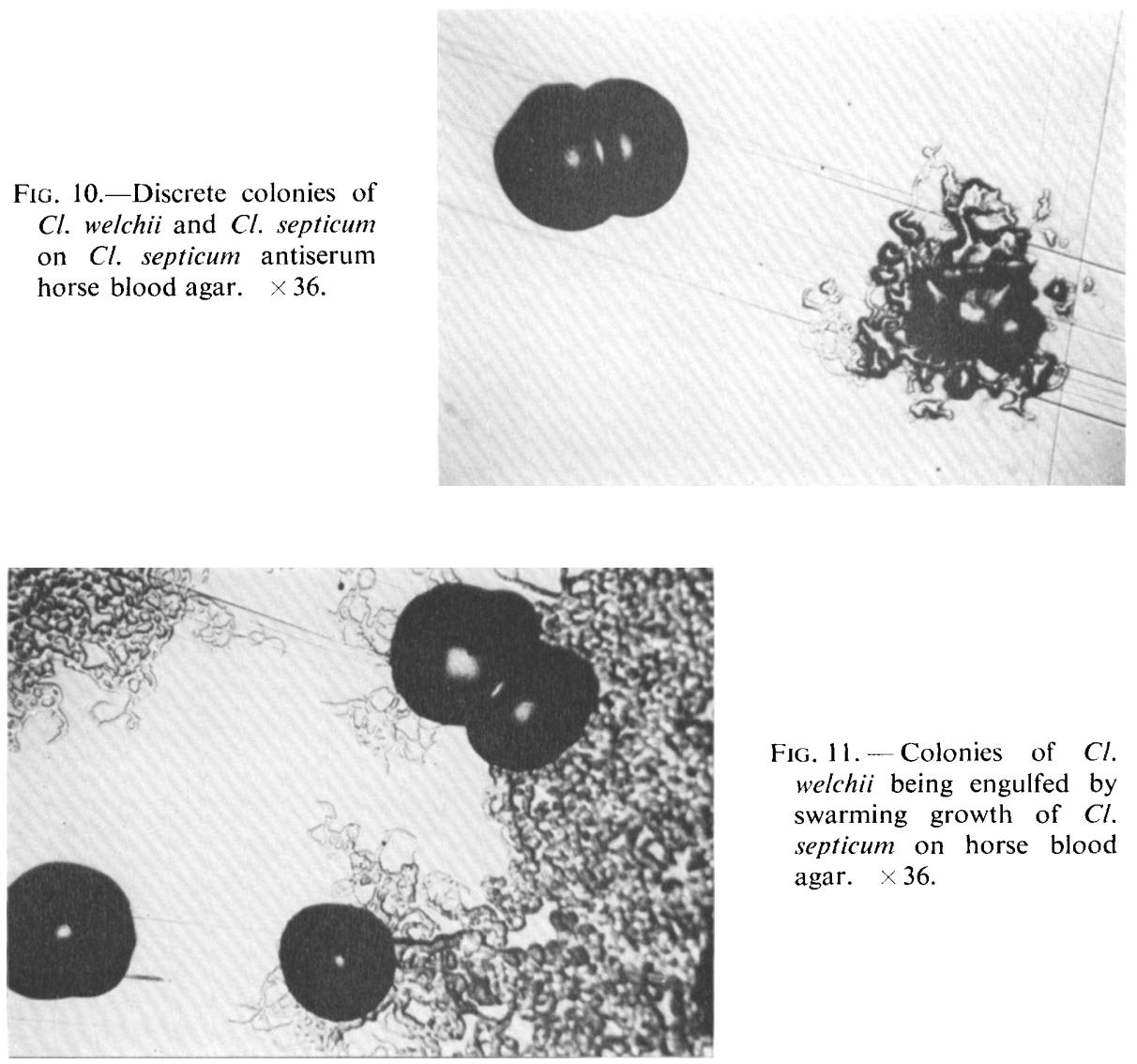

FIG. 11.-Colonies of $\mathrm{Cl}$. welchii being engulfed by swarming growth of $\mathrm{Cl}$. septicum on horse blood agar. $\times 36$. 
All strains of $\mathrm{Cl}$. septicum gave countable colonies on antiserum blood agar (fig. 3). The colonies were small, circular and discrete, with a mildly lobulated edge and usually an absence of rhizoidal outgrowth. On firm blood agar, on the other hand, two of the strains produced growth that was so irregular in structure that it was not possible to identify separate colonies accurately and counts could not be obtained. Colonies of the other eight strains were markedly irregular but were easily countable (figs. 4 and 5).

Among the eight strains for which surface viable counts were obtained on both media, seven showed essentially comparable results, and the remaining one gave a count that was marginally higher on the firm agar medium.

\section{Isolation of Clostridium chauvoei from mixtures with Cl. septicum on Cl. septicum antiserum blood agar}

The principle of inhibition of swarming growth of $\mathrm{Cl}$. septicum by antiserum was applied to the separation of two strains of Cl. chauvoei (nos. NCTC8070 and 8590) from mixtures with $\mathrm{Cl}$. septicum in plate cultures. Mixtures of the two species were examined on half-antiserum horse blood agar plates enriched with glucose and liver extract. It was a simple matter to pick off discrete colonies of $\mathrm{Cl}$. chauvoei from the antiserum half of the plates; the untreated half of each plate showed a confluent growth of $\mathrm{Cl}$. septicum overlying colonies of Cl. chauvoei (figs. 6 and 7).

The use of antiserum-treated plates was equally successful in the separation of other anaerobes from mixtures with $\mathrm{Cl}$. septicum, including $\mathrm{Cl}$. welchii type A, $\mathrm{Cl}$. bifermentans, $\mathrm{Cl}$. oedematiens type A and $\mathrm{Cl}$. histolyticum (figs. 8-11). Unlike concentrated agar media, antiserum-treated plates had no adverse effect on the growth of other clostridia, but allowed the free development of their characteristic colonies. This was especially marked in the case of $\mathrm{Cl}$. chauvoei, which may grow poorly or not at all on firm agar media.

\section{Discussion}

The formation by $\mathrm{Cl}$. septicum of discrete surface colonies in the presence of $\mathrm{Cl}$. septicum $\mathrm{O}$ antiserum is probably the result of "agglutination" of the growing culture. Watt (1972) made use of this procedure for the enumeration of $\mathrm{Cl}$. septicum and obtained useful inhibition with a selected antitoxic serum. It is important to note that the definite results obtained in our present study with many strains of $\mathrm{Cl}$. septicum are presumably attributable to the use of a polyvalent serum intentionally prepared against the $\mathrm{O}$ antigenic components of the organism.

Although the use of firm agar is almost as effective as the use of plates bearing $\mathrm{Cl}$. septicum antiserum in preventing surface swarming growth of $\mathrm{Cl}$. septicum, it is less satisfactory as a method for the isolation of other clostridia from mixtures with $\mathrm{Cl}$. septicum. This applies particularly to exacting species such as $\mathrm{Cl}$. chauvoei, the growth of which may be so retarded by high concentrations of agar that macroscopic colonies fail to develop. 
It seems that the antiserum method of restricting surface growth of $\mathrm{Cl}$. septicum may find a useful application in veterinary anaerobic bacteriology. Cl. septicum is a normal inhabitant of the intestinal tract of herbivorous animals, whence it rapidly invades the tissues post mortem. Consequently, the isolation of $\mathrm{Cl}$. septicum from these animals does not imply a causal relation to a pathological process. In the case of isolation of $\mathrm{Cl}$. chauvoei from blackleg lesions, difficulty is often encountered when $C l$. chauvoei is overgrown by the nutritionally less demanding $\mathrm{Cl}$. septicum, a state of affairs that is aggravated in primary plate cultures by the swarming habit of $\mathrm{Cl}$. septicum. Batty and Walker (1963) utilised the dissimilarity of the $\mathrm{O}$ antigenic components of $\mathrm{Cl}$. septicum and $C l$. chauvoei to develop a fluorescent antibody-staining technique that serves to differentiate between the two species in smears and tissue sections. We have extended this exploitation of the property of antigenic dissimilarity to the separation of $\mathrm{Cl}$. chauvoei from $\mathrm{Cl}$. septicum in artificial culture.

Surface viable counts of $\mathrm{Cl}$. septicum may be conveniently obtained by the method of Miles and Misra when horse blood agar treated with $\mathrm{Cl}$. septicum $\mathrm{O}$ antiserum is used as the plating medium. Antiserum-treated agar is marginally superior to firm agar for this purpose.

\section{SUMMARY}

A $\mathrm{Cl}$. septicum $\mathrm{O}$ antiserum, prepared in the rabbit against the two serological groups of Moussa, inhibits the swarming growth of $\mathrm{Cl}$. septicum in plate cultures. Advantage may be taken of this in the separation of other organisms from mixtures with $\mathrm{Cl}$. septicum and in performing surface viable counts with Cl. septicum.

We are greatly indebted to $\mathrm{Mr}$ J. Harrison for production of the photographs.

\section{REFERENCES}

Batty, Irene, AND Walker, P. D. 1963. Differentiation of Clostridium septicum and Clostridium chauvoei by the use of fluorescent labelled antibodies. J. Path. Bact., 85, 517. Cruickshank, R. 1965. Medical microbiology, 11th ed., Edinburgh. p. 742.

Miles, A. A., MisRa, S. S., AND Irwin, J. O. 1938. The estimation of the bactericidal power of the blood. J. Hyg., Camb., 38, 732.

Moussa, R. S. 1959. Antigenic formulae for Clostridium septicum and Clostridium chauvoei. J. Path. Bact., 77, 341.

WATT, B. 1972. The recovery of clinically important anaerobes on solid media. J. Med. Microbiol., 5, 211.

Williams, Kathleen 1971. Some observations on Clostridium tetani. Med. Lab. Technol., 28, 399.

Williams, KathleEN, AND Willis, A. T. 1970. A method of performing surface viable counts with Clostridium tetani. J. Med. Microbiol., 3, 639.

Willis, A. T., AND Williams, KathleEn 1970. Some cultural reactions of Clostridium tetani. J. Med. Microbiol., 3, 291. 